\title{
COMPATIBILIDADE DE METARHIZIUM ANISOPLIAE COM CARRAPATICIDAS QUÍMICOS
}

\section{F.B. Soares; A.C. Monteiro}

Universidade Estadual Paulista, Faculdade de Ciências Agrárias e Veterinárias, Via de Acesso Prof. Paulo Donato Castellane, s/nº, CEP 14884-900, Jaboticabal, SP, Brasil. E-mail: montecar@fcav.unesp.br

\section{RESUMO}

A associação de agentes biológicos e químicos pode ser uma estratégia promissora para o controle de insetos e ácaros, visando a aumentar a eficiência, reduzir custos e impactos ambientais. Este trabalho objetivou avaliar a compatibilidade do fungo Metarhizium anisopliae (Metsch.) Sorokin com carrapaticidas químicos atualmente usados no controle de Riphicephalus (Boophilus) microplus (Canestrini). O fungo foi cultivado em meio contendo 40, 55, 70, 85 e 100\% da dose recomendada (DR) de carrapaticidas comerciais contendo vários princípios ativos, formulados em diferentes composições. Avaliou-se o crescimento vegetativo, a esporulação e a viabilidade do fungo e fez-se a classificação toxicológica dos acaricidas. Os carrapaticidas contendo clorfenvinfós + diclorvós $\left(\right.$ Amiphós $\left.^{\circledR}\right)$, cipermetrina + clorpirifós + citronelal $\left(\right.$ Barrage $^{\circledR}$ ) e amitraz + clorpirifós (Carbeson ${ }^{\circledR}$ ) foram classificados como tóxicos, pois inibiram o crescimento e a esporulação, e apenas o último não afetou a viabilidade do fungo. O carrapaticida contendo cipermetrina $\left(\right.$ Colosso $\left.^{\circledR}\right)$ foi classificado como compatível e moderadamente compatível nas doses de 40 e $65 \%$ da DR, respectivamente, e aquele contendo spinosad (Elector ${ }^{\circledR}$ ) foi o que menos afetou os parâmetros avaliados, sendo moderadamente compatível nas doses de 55 a 100\% da DR e compatível na dose de $40 \%$ da DR. A maioria dos carrapaticidas químicos avaliados não foi compatível com o fungo M. anisopliae; apenas aqueles formulados com cipermetrina e com spinosad mostraram alguma compatibilidade para serem empregados em uma possível estratégia de uso associado.

PALAVRAS-CHAVE: Controle biológico, controle microbiano, fungo entomopatogênico, Riphicephalus (Boophilus) microplus, carrapato de bovinos.

\section{ABSTRACT}

COMPATIBILITY OF METARHIZIUM ANISOPLIAE WITH CHEMICAL ACARICIDES. The combined use of chemical and biological control agents could prove to be a successful strategy against insects and ticks, which could improve the efficiency attained by conventional strategies and also lessen environmental impact that could be caused by these substances. The present study assessed compatibility of the entomopathogenic fungus Metarhizium anisopliae (Metsch.) Sorokin to chemical substances currently used for controlling Riphicephalus (Boophilus) microplus (Canestrini), to verify compatibility of these for possible associated use. The fungus was cultivated in a medium to which was incorporated 40,55,70, 85 and $100 \%$ of the recommended dosage (RD) of commercial acaricides containing various active ingredients formulated in different compositions. To verify compatibility, fungus vegetative growth, spore production and viability were assessed, and all acaricides were classified regarding toxicity. The chemical acaricides containing chlorfenvinphos + dichlorvos $\left(\right.$ Amiphós $^{\circledR}$ ), cipermetrin + chlorpiriphos + citronelal $\left(\right.$ Barrage $^{\circledR}$ ) and amitraz + chlorpiriphos (Carbeson ${ }^{\circledR}$ ) were classified as toxic after causing inhibition of fungal growth and spore production, the last one being the only agent that did not affect fungal viability. The acaricide containing cipermetrin $\left(\right.$ Colosso $\left.^{\circledR}\right)$ was classified as compatible and moderately compatible in 40 and $65 \%$ of the RD, respectively, and the acaricide containing spinosad (Elector ${ }^{\circledR}$ ) was the agent that least affected the factors evaluated, being classified as moderately compatible at 55 to $100 \%$ of the RD, and as compatible at $40 \%$ of RD. Most of the chemical acaricides evaluated were not compatible with $M$. anisopliae. The acaricide formulated with cipermetrin and spinosad was the only one that showed some compatibility for use in an integrated pest management.

KEY WORDS: Biological control, microbial control, entomopathogenic fungus, Riphicephalus (Boophilus) microplus, cattle tick. 


\section{INTRODUÇÃO}

Rhipicephalus (Boophilus) microplus (Canestrini) é um ectoparasita bovino responsável por significativas perdas econômicas em rebanhos de áreas tropicais e subtropicais, devido ao parasitismo que reduz a produção de leite e bezerros, além de ocasionar gastos na aquisição de produtos para seu controle (CASTRO; NEWSON, 1993). Há ainda o estresse causado aos animais para controle do parasito e a redução da taxa de natalidade, além de serem considerados importantes transmissores de patógenos, como Babesia, Ehrlichia e Anaplasma, que muitas vezes levam os animais a óbito (MonTEIRO et al., 2003). Segundo GRISI et al. (2002), o Brasil tem prejuízos superiores a um bilhão de dólares anuais com esse parasita.

Atualmente, o controle do parasita é feito principalmente com o uso de carrapaticidas químicos, cuja efetividade tem diminuído devido ao aumento da resistência dos carrapatosa esses produtos (SAMISHetal., 2001). Uma dasalternativas aouso doscarrapaticidaséa utilizaçãodemicro-organismos, principalmentefungos ebactérias, potenciaiscontroladores decarrapatos (ZHIOUA et al., 1999). Algumas espécies de fungos já foram isoladas de todos os estágios de desenvolvimento de carrapatos, inclusive os ovos. Além disso, a utilização dessesmicro-organismos para controlebiológicoreduz a aquisição de resistência pelas pragas.

De acordo com LOURENÇÃo et al. (1993), para o controle de pragas na agricultura, os fungos podem ser usados de forma isolada ou em associação com outros métodos, como os inseticidas químicos (OliveIRA et al., 2002; ANDALÓetal., 2004; WeNZELet al., 2004) ou naturais de origem vegetal (MARQUES et al., 2004). Nesses trabalhos, foi demonstrado quealguns dessesinseticidas são compatíveis comfungosentomopatogênicos, podendo ser utilizados concomitantemente. Na pecuária, o uso associado também pode ser uma estratégia viável, diminuindo gastos, a possibilidade de seleção de pragas e doenças resistentes aos produtos químicos, a intoxicação do rebanho e dos trabalhadores que aplicam os produtos e os resíduos destes, tanto na carne e no leite consumidos, quanto no ambiente.
No entanto, poucos estudos foram realizados para se determinar a compatibilidade entre fungos patogênicos de carrapatos e carrapaticidas químicos (PAĨ̃O, 2000; BAHIENSIS; BITTENCOURT, 2004; ER; GÖKÇE, 2004; BARCI, 2007), apesar dos benefícios do controle associado. Assim sendo, este trabalho teve por objetivo avaliar a compatibilidade do fungo $M$. anisopliae (Метsсн.) SoroKIn com carrapaticidas químicos atualmente usados no controle de $R$. (B.) microplus, para um possível uso associado entre esses agentes.

\section{MATERIAL E MÉTODOS}

Foi utilizado o isolado E9 de M. anisopliae obtido de Deois flavopicta Stal. O isolado foi cultivado em placas de Petri contendo meio de batata, dextrose e ágar (BDA) mantidas em estufa a $27 \pm 1^{\circ} \mathrm{C}$, em ausência de luz, durante 12 dias.

Foram utilizados cinco carrapaticidas químicos comerciais compostos de vários princípios ativos, formulados em diferentes composições, empregados em volumes adequados para obter as doses desejadas e preparados seguindo prescrição dos seus fabricantes (Tabela 1).

Os carrapaticidas foram utilizados nas seguintes doses (em \% da doserecomendada pelofabricante): 40 , $55,70,85 \mathrm{e} 100 \%$, tendosidoadicionadosaomeioemque o fungo foi cultivado na temperatura entre 45 e $50^{\circ} \mathrm{C}$, para evitar alteração das propriedades dos compostos químicosavaliados. Umdoscarrapaticidas apresentava os princípios ativos separadamente, na forma de um sache contendo $10 \mathrm{mg}$ de amitraz $50 \%$ e um frasco contendo $20 \mathrm{~mL}$ de clorpirifós $50 \%$. Calcularam-se as quantidades dos princípios ativos a seremusadas. Pelo fato de serem quantidades muito pequenas em função do pequeno volume de meio preparado, quantidades apropriadas dos princípios ativos foram diluídas em $10 \mathrm{~mL}$ de água destilada esterilizada, obtendo-se, assim, uma solução cuja concentração por mililitro era a desejada para se conseguir a dose recomendada. Para obtenção das demais dosagens foram retiradas alíquotas dessa solução, com auxílio de pipeta automática.

Tabela 1 - Carrapaticidas químicos utilizados nos ensaios com os respectivos princípios ativos, grupos químicos, concentração, dose recomendada e administração.

\begin{tabular}{|c|c|c|c|c|}
\hline Princípio ativo e nome comercial & Grupo químico & $\begin{array}{c}\text { Concentração } \\
(\mathrm{g} / \mathrm{L})\end{array}$ & $\begin{array}{c}\text { Dose recomendada } \\
(\mathrm{L} / \mathrm{L})\end{array}$ & Administração \\
\hline $\begin{array}{l}\text { Amitraz + Clorpirifós } \\
\left(\text { Amiphós }{ }^{\circledR}\right)\end{array}$ & $\begin{array}{l}\text { Formamidina e } \\
\text { Organofosforado }\end{array}$ & $\mathrm{ND}^{1}$ & $\mathrm{ND}^{1}$ & Pulverização \\
\hline Cipermetrina $\left(\right.$ Barrage $^{\circledR}$ ) & Piretroide & 150 & $1 / 1000$ & Pulverização \\
\hline Diclorvós + Clorfenvinfós (Carbeson ${ }^{\circledR}$ ) & Organofosforado & $600+200$ & $1 / 400$ & Pulverização \\
\hline $\begin{array}{c}\text { Cipermetrina + Clorpirifós + } \\
\text { Citronelal }\left(\text { Colosso }^{\circledR}\right)\end{array}$ & $\begin{array}{c}\text { Piretroide e } \\
\text { Organofosforado }\end{array}$ & $150+250+10$ & $1 / 800$ & Pulverização \\
\hline Spinosad (Elector $\left.{ }^{\circledR}\right)$ & Espinosinas & 25 & $1 / 100$ & Pulverização \\
\hline
\end{tabular}

${ }^{1}$ Não determinado: Princípios ativos apresentados separadamente, na forma de um sachê contendo $10 \mathrm{mg}$ de Amitraz $50 \%$ e um frasco contendo $20 \mathrm{~mL}$ de Clorpirifós 50\%, para diluição em 20 L de água. 
Os parâmetros de avaliação do desempenho do fungo em relação às doses de carrapaticida foram: crescimento vegetativo, esporulaçãoe viabilidadedos conídios. O crescimento das colônias foi quantificado por meio de medidas, em milímetros, de três diâmetros marcados na parte externa do fundo da placa de Petri. As medidas foram efetuadas a cada três dias, do 3 o ao 15o dias após a inoculação. Cada placa correspondeu a uma repetição e, para cada concentração de carrapaticida (tratamento), foram feitas 5 placas.

A produção de conídios foi avaliada coletandose uma amostra do centro, uma mediana e uma da periferia de cada colônia com auxílio de um anel metálico esterilizado com $8 \mathrm{~mm}$ de diâmetro no 15 o dia de incubação. Para cada tratamento, foram coletadas amostras de três colônias (repetições). As amostras foram transferidas individualmente para tubos de ensaio com $10 \mathrm{~mL}$ de uma solução 1:1 de $\mathrm{NaCl}(0,089 \% \mathrm{p} / \mathrm{v})$ e Tween $80^{\circledR}(0,1 \% \mathrm{v} / \mathrm{v})$ esterilizada. Após a remoção dos conídios por vigorosa agitação em agitador elétrico de tubos, foi feita, para cada tubo, uma contagem ao microscópio óptico em câmara de Neubauer, utilizando-se diluição da suspensão quando necessário.

A viabilidade dos conídios foi avaliada por meio de cultivo e exame direto em lâmina ao microscópio. Para tanto, lâminas demicroscopia esterilizadas foram recobertas com uma fina camada de BDA contendo os diferentes carrapaticidas químicos nas concentrações já mencionadas. Na parte inferior de cada lâmina foram marcados três pontos e sobre o meio de cultura foi inoculado, em cada ponto, $0,1 \mathrm{~mL}$ de suspensão fúngica com $10^{5} \mathrm{con}$. $\mathrm{mL}^{-1}$. As lâminas foram incubadas a $27 \pm 1^{\circ} \mathrm{C}$, em ausência de luz, durante 15 horas. Em seguida, foram observados 150 conídios em cada ponto da lâmina, entre germinados enãogerminados, sendo estabelecida uma porcentagem. Cada lâmina correspondeu a uma repetição, e para cada tratamento foram feitas três lâminas.

Os ensaios foram realizados no delineamento inteiramente casualizado. Os dados foram submetidos à análise de variância pelo teste $\mathrm{F}$, e as médias foram comparadas pelo teste de Tukey, a 5\% de probabilidade pelo programa ESTAT (1997).

Para determinação do efeito tóxico dos carrapaticidas foi utilizada a fórmula proposta por ALVES et al. (2007), utilizando os parâmetros crescimento vegetativo, esporulação e viabilidade:

$$
\mathrm{IB}=\frac{47[C V]+43[E S P]+10[G E R]}{}
$$

em que:

I.B. = Índice Biológico; CV: porcentagem de crescimento vegetativo da colônia após 15 dias em relação à testemunha; ESP: porcentagem de esporulação após 15 dias em relação à testemunha; GER: porcentagem de germinação dos conídios após 15 horas em relação à testemunha. Não foram utilizadas casas decimais para o cálculo do I.B.

Com os valores obtidos de I.B., fez-se a classificação toxicológica dos carrapaticidas nas diferentes dosagens, de acordo escala estabelecida por ALves et al. (2007): de 0 a 41: tóxico; de 42 a 66: moderadamente compatível; > 66: compatível.

\section{RESULTADOS E DISCUSSÃO}

Ocarrapaticida à base declorfenvinfós + diclorvós foi o único que inibiu totalmente o crescimento das colônias (Fig. 1A). Esse resultado está de acordo com o obtido por BARCI (2007), que utilizou dois carrapaticidas contendo diclorvós e não verificou crescimento vegetativo de Beauveria bassiana (BALs.) Vuill.Segundo GHINI; KIMATI (2000), esse fato pode ser explicado pela ação do organofosforado (diclorvós) que, nos fungos fitopatogênicos, interfere na produção de parede celular. Isso ocorre por meio da ação desse composto sobre a enzima que converte fosfatidiletanolamina para fosfatidilcolina e inibe, dessa maneira, a síntese de quitina, componente da parede celular.

Para o carrapaticida formulado com amitraz + clorpirifós, a inibição aumentou com o aumento das dosagens (Fig. 1B), efeito também observado por PAIÃO (2000), que cultivou M. anisopliae e B. bassiana em meio contendovárias doses deamitraz. Esseachadotambém foi observado com o carrapaticida formulado com clorpirifós que, a partir de $40 \%$ da DR, inibiu o crescimento, mas não houve diferença entre as dosagens de 55a 100\% (Fig. 1C). Ocarrapaticida contendo spinosad inibiu o crescimento a partir de $40 \%$ da DR, mas não houve diferença no crescimento obtido nas doses de 40 e $55 \%$ (Fig. 1D). No meio em que foi adicionado o carrapaticidaà base decipermetrina, a inibição docrescimento ocorreu a partir de $70 \%$ da DR, não havendo inibição nas menores dosagens (Fig. 1E). O resultado é semelhante ao obtido por ER; GÖRKÇE (2004), os quais utilizaram $1 / 10$ da doserecomendada decipermetrina e não verificaram inibição do crescimento do fungo Paecilomyces fumosoroseus Wize.

Todos os carrapaticidas inibiram a esporulação, confirmandooocorridocomocrescimento vegetativo (Tabela 2). Para o carrapaticida à base declorfenvinfós + diclorvós, por não haver crescimento de colônia, não houve esporulação. Os carrapaticidas contendo amitraz + clorpirifós e cipermetrina + clorpirifós + citronelal inibiram a esporulação a partir de $40 \%$ da DR. OliveIRA et al. (2002) utilizaram subdosagens de um carrapaticida contendo amitraz e verificaram que houve redução significativa da esporulação de M. anisopliae. PACHAMUthu et al. (1999) cultivaram M. anisopliae em 500ppm de clorpirifós e não houve esporulação. Para os carrapaticidas formulados à base 
de cipermetrina e de spinosad, a dose de $40 \%$ da DR foi a que menos influenciou a esporulação e, a partir de $70 \%$ da DR docarrapaticidaà base decipermetrina, houve grande diminuição da produção de conídios. O carrapaticida contendo spinosad foi o que causou a menor inibição da esporulação (Tabela 2).

Tabela 2 - Produção de conídios de Metarhizium anisopliae cultivado em meio contendo diferentes doses dos carrapaticidas químicos após 15 horas de incubação, a $27^{\circ} \mathrm{C}$.

\begin{tabular}{lccccc}
\hline \multirow{2}{*}{$\begin{array}{c}\text { Dose }(\% \\
\text { da D.R.) }\end{array}$} & $\begin{array}{c}\text { Clorfenvinvós }+ \\
\text { Diclorvós }\end{array}$ & $\begin{array}{c}\text { Amitraz }+ \\
\text { Clorpirifós }\end{array}$ & $\begin{array}{c}\text { Cipermetrina }+ \\
\text { Clorpirifós + Citronelal }\end{array}$ & Cipermetrina & Spinosad \\
\cline { 2 - 6 } Controle & $46,97 \mathrm{a}$ & $45,45 \mathrm{a}$ & $36,65 \mathrm{a}$ & $67,20 \mathrm{a}$ & $46,30 \mathrm{a}$ \\
40 & $0,00 \mathrm{~b}$ & $1,32 \mathrm{~b}$ & $5,82 \mathrm{~b}$ & $41,95 \mathrm{~b}$ & $28,55 \mathrm{~b}$ \\
55 & $0,00 \mathrm{~b}$ & $1,73 \mathrm{~b}$ & $3,86 \mathrm{~b}$ & $34,37 \mathrm{c}$ & $22,60 \mathrm{c}$ \\
70 & $0,00 \mathrm{~b}$ & $0,46 \mathrm{~b}$ & $3,85 \mathrm{~b}$ & $1,63 \mathrm{~d}$ & $19,12 \mathrm{~cd}$ \\
85 & $0,00 \mathrm{~b}$ & $0,54 \mathrm{~b}$ & $4,37 \mathrm{~b}$ & $1,55 \mathrm{~d}$ & $15,92 \mathrm{~d}$ \\
100 & $0,00 \mathrm{~b}$ & $1,04 \mathrm{~b}$ & $3,01 \mathrm{~b}$ & $1,29 \mathrm{~d}$ & $15,77 \mathrm{~d}$ \\
\hline F & $561,53^{* *}$ & $491,9^{* *}$ & $314,77^{* *}$ & $746,51^{* *}$ & $89,95^{* *}$ \\
C.V. (\%) & 3,7 & 9,9 & 4,8 & 5,02 & 2,7 \\
\hline
\end{tabular}

Valores originais, mas análise realizada com dados transformados em log $(x+1)$.

Médias seguidas da mesma letra na coluna não diferem entre si pelo teste de Tukey a $5 \%$ de probabilidade.

D.R.: dose recomendada pelo fabricante.

C.V. Coeficiente de variação.

**Significativo a $1 \%$ de probabilidade pelo teste F.

Tabela 3 - Viabilidade de Metarhizium anisopliae cultivado em meio contendo diferentes doses dos carrapaticidas químicos após 15 horas de incubação, a $27^{\circ} \mathrm{C}$.

\begin{tabular}{lccccc}
\hline \multirow{2}{*}{$\begin{array}{c}\text { Dose (\% } \\
\text { da D.R.) }\end{array}$} & $\begin{array}{c}\text { Clorfenvinfós }+ \\
\text { Diclorvós }\end{array}$ & $\begin{array}{c}\text { Amitraz }+ \\
\text { Clorpirifós }\end{array}$ & $\begin{array}{c}\text { Cipermetrina + Clorpirifós } \\
+ \text { Citronelal }\end{array}$ & $\begin{array}{c}\text { Cipermetrina } \\
\text { Spinosad }\end{array}$ \\
\cline { 2 - 6 } Controle & $89,20 \mathrm{a}$ & $91,16 \mathrm{a}$ & $99,86 \mathrm{a}$ & $97,26 \mathrm{a}$ & $95,26 \mathrm{a}$ \\
40 & $0,00 \mathrm{~b}$ & $88,33 \mathrm{a}$ & $97,06 \mathrm{~b}$ & $95,80 \mathrm{a}$ & $90,20 \mathrm{ab}$ \\
55 & $0,00 \mathrm{~b}$ & $87,66 \mathrm{a}$ & $93,06 \mathrm{bc}$ & $97,80 \mathrm{a}$ & $85,53 \mathrm{~b}$ \\
70 & $0,00 \mathrm{~b}$ & $85,00 \mathrm{a}$ & $91,00 \mathrm{c}$ & $94,86 \mathrm{a}$ & $83,20 \mathrm{~b}$ \\
85 & $0,00 \mathrm{~b}$ & $89,44 \mathrm{a}$ & $94,53 \mathrm{bc}$ & $94,06 \mathrm{a}$ & $6,60 \mathrm{c}$ \\
100 & $0,00 \mathrm{~b}$ & $88,33 \mathrm{a}$ & $96,53 \mathrm{bc}$ & $78,53 \mathrm{~b}$ & $5,46 \mathrm{c}$ \\
\hline F & $262,77^{* *}$ & $2,62^{\text {n.s. }}$ & $5,35^{* *}$ & $11,46^{* *}$ & $279,72^{* *}$ \\
\hline C.V. $(\%)$ & 2,12 & 2,91 & 5,11 & 4,76 & 5,77 \\
\hline
\end{tabular}

Valores originais, mas análise realizada com dados transformados em arc seno

Médias seguidas da mesma letra na coluna não diferem entre si pelo teste de Tukey, a 5\% de probabilidade

D.R.: dose recomendada pelo fabricante

C.V.: Coeficiente de variação

n.s. não significativo

** Significativo a $1 \%$ de probabilidade pelo teste $\mathrm{F}$

Tabela 4 - Valores do Índice Biológico (I.B.) e classificação dos carrapaticidas químicos quanto à toxicidade ao fungo Metarhizium anisopliae nas diferentes dosagens.

\begin{tabular}{|c|c|c|c|c|c|c|c|c|c|c|}
\hline \multirow{2}{*}{$\begin{array}{l}\text { Dose } \\
\text { (\% da } \\
\text { D.R.) }\end{array}$} & \multicolumn{2}{|c|}{$\begin{array}{c}\text { Clorfenvinfós + } \\
\text { Diclorvós }\end{array}$} & \multicolumn{2}{|c|}{$\begin{array}{c}\text { Cipermetrina } \\
+ \text { Clorpirifós + } \\
\text { Citronelal }\end{array}$} & \multicolumn{2}{|c|}{$\begin{array}{l}\text { Amitraz + } \\
\text { Clorpirifós }\end{array}$} & \multicolumn{2}{|c|}{ Cipermetrina } & \multicolumn{2}{|c|}{ Spinosad } \\
\hline & I.B. & Toxicidade & I.B. & Toxicidade & I.B. & Toxicidade & I.B. & Toxicidade & I.B. & Toxicidade \\
\hline 40 & 0 & $\mathrm{~T}$ & 32 & $\mathrm{~T}$ & 25 & $\mathrm{~T}$ & 73 & $\mathrm{C}$ & 74 & $\mathrm{C}$ \\
\hline 55 & 0 & $\mathrm{~T}$ & 26 & $\mathrm{~T}$ & 23 & $\mathrm{~T}$ & 65 & MC & 65 & $\mathrm{MC}$ \\
\hline 70 & 0 & $\mathrm{~T}$ & 27 & $\mathrm{~T}$ & 20 & $\mathrm{~T}$ & 26 & $\mathrm{~T}$ & 60 & $\mathrm{MC}$ \\
\hline 85 & 0 & $\mathrm{~T}$ & 28 & $\mathrm{~T}$ & 20 & $\mathrm{~T}$ & 26 & $\mathrm{~T}$ & 48 & $\mathrm{MC}$ \\
\hline 100 & 0 & $\mathrm{~T}$ & 25 & $\mathrm{~T}$ & 20 & $\mathrm{~T}$ & 24 & $\mathrm{~T}$ & 62 & $\mathrm{MC}$ \\
\hline
\end{tabular}

T:Tóxico; MC: Moderadamente compatível; C: Compatível 

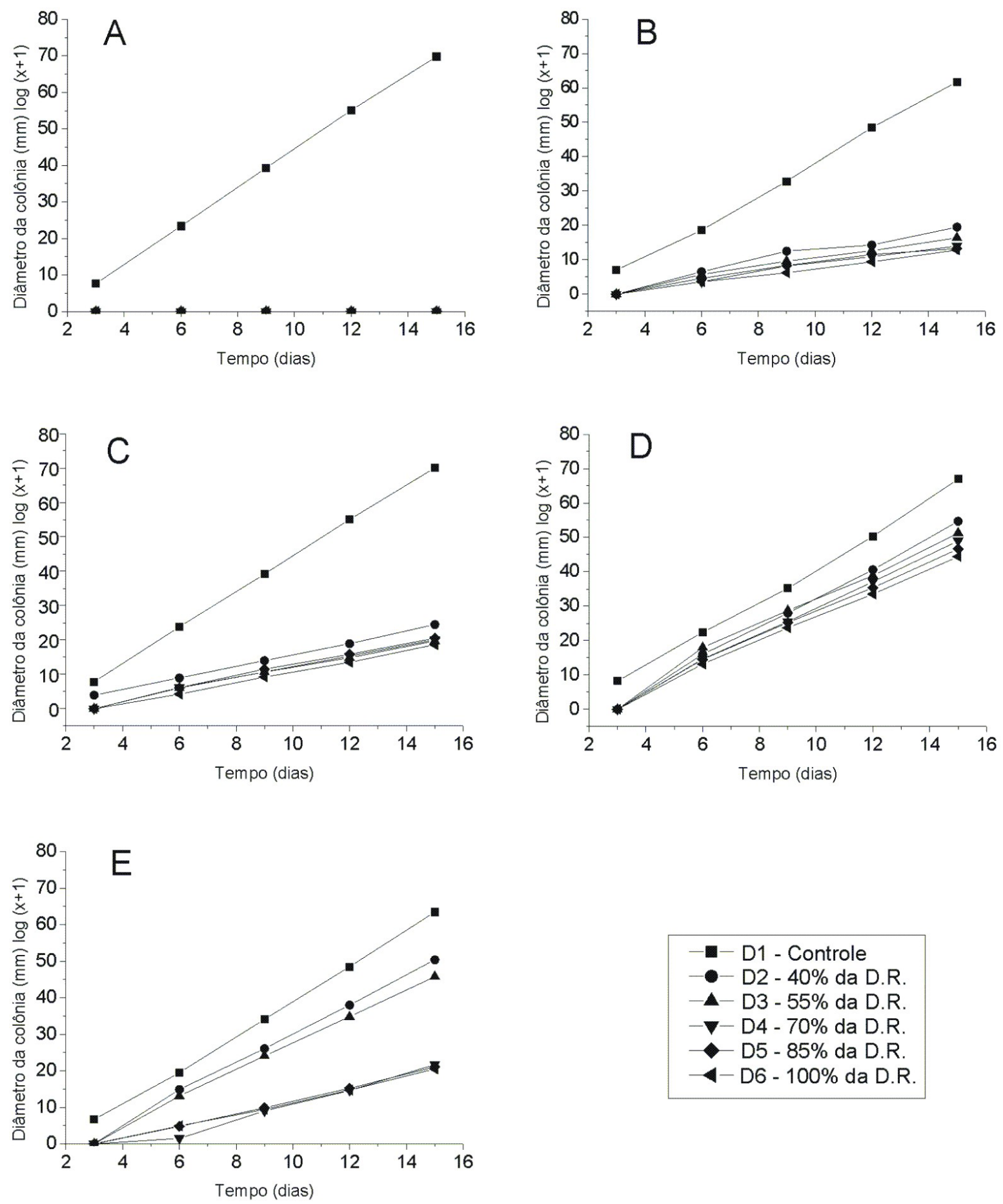

Fig. 1 - Crescimento radial do isolado E9 de Metarhizium anisopliae e meio de BDA contendo diferentes doses dos carrapaticidas químicos compostos por diversos principios ativos formulados em diferentes composições. A: diclorvós + clorfenvinfós; B: amitraz + clorpirifós; C: cipermetrina + clorpirifós + citrobelal; D: spinosad; E: cipermetrina.

A viabilidade dos conídios foi completamente afetada pelo carrapaticida à base de clorfenvinfós + diclorvós em todas as doses utilizadas. Para o carrapaticida contendo spinosad, a viabilidade não diferiu da testemunha apenas na dose de $40 \%$ da DR e houve um incremento da inibição da germi- nação dos conídios com o aumento das doses. $\mathrm{O}$ carrapaticida formulado com cipermetrina + clorpirifós + citronelal ocasionou pequena redução da germinação dos conídios em relação à testemunha, mas em todas as dosagens a viabilidade foi maior que $90 \%$ (Tabela 3). 
Os carrapaticidas à base de cipermetrina (exceto na dose recomendada) e de amitraz + clorpirifós não inibiram a viabilidade nas diferentes dosagens (Tabela 3). Segundo PAĩ̃o (2000), amitraz usado em até $80 \%$ da D.R. não inibiu a viabilidade de $M$. anisopliae. De acordo com Pachamuthu et al. (1999), os fatores que atuam sobre a germinação não devem ser os mesmos que atuam sobre a esporulação e o crescimento, quando em contato com inseticidas. Para o controle associado, esse resultado é importante, pois, se utilizadas subdosagens, a germinação dos conídios, que é o evento necessário para que o fungo inicie a penetração no hospedeiro, não será afetada.

Por meio do cálculo do Índice Biológico, determinou-se a toxicidade dos carrapaticidas para $M$. anisopliae (Tabela 4). Os carrapaticidas à base de clorfenvinfós + diclorvós, de amitraz + clorpirifóse de cipermetrina + clorpirifós + citronelal foram tóxicos ao fungo em todas as doses utilizadas, enquanto o carrapaticida à base de cipermetrina foi classificado como compatível na dose de $40 \%$ da DR e moderadamente compatível na dose de $55 \%$. Ocarrapaticida formulado com spinosad foi considerado compatível na dose de $40 \%$ da D.R. emoderadamente compatível nas demais dosagens. No entanto, nessas dosagens a viabilidade dos conídios foi afetada, e este é um aspecto negativo para o uso associado.

A compatibilidade do carrapaticida à base de spinosad com $M$. anisopliae pode ser devida à origem natural dos ingredientes ativos, que provém de fermentação realizada pela bactéria actinomiceta Saccharopolyspora spinosa Mertz; Yao. De acordo com Cleveland (2007), há várias documentações sobre a segurança ambiental do produto, que possui baixa toxicidade aos mamíferos e aos organismos não-alvo e rápida degradação no ambiente.

A ação patogênica de $M$. anisopliae associada à subdosagens dos carrapaticidas químicos pode ser uma estratégia viável no controle de $R$. (B.) microplus, promovendo a morte do ácaro com redução dos efeitos tóxicos e impactos ambientais causados pelo uso dos carrapaticidas. Entretanto, diversos aspectos desse uso associado precisam ser melhor investigados, principalmente em condições de campo.

\section{CONCLUSÕES}

A maioria dos carrapaticidas químicos avaliados não se mostrou compatível com M. anisopliae, sendo o crescimento vegetativo e a esporulação do fungo os parâmetros mais afetados, enquanto a viabilidade foi pouco afetada. As dosagens utilizadas tiveram pequena influência na ação tóxica, e apenas os carrapaticidas formulados com cipermetrina e com spinosad mostraram alguma compatibilidade para serem empregados em uma possível estratégia de uso associado com o fungo.

\section{REFERÊNCIAS}

ANDALÓ, V.; MOINO JUNIOR, A.; SANTA-CECÍLIA, L.V.C.; SOUZA, G.C. Compatibility of Beauveria bassiana with chemical pesticides for the control of the coffee root mealybug Dysmococcus texensis Tinsley (Hemiptera: Pseudococcidae). Neotropical Entomology, v.33, n.4, p.463-467, 2004.

ALVES, S.B.; HADDAD, M.L.; FAION, M.; BAPTISTA, G.C. de; ROSSI-ZALAF, L.S. Novo índice biológico para classificação da toxicidade de agrotóxicos para fungos entomopatogênicos. In: SIMPÓSIO DE CONTROLE BIOLÓGICO, 10., 2007, Brasília. Anais. Brasília: Embrapa-SEB, 2007. 1CD-ROM.

BAHIENSIS, T.C.; BITTENCOURT, V.R.E.P. Laboratory evaluation of the compatibility and the synergism between the entomopathogenic fungus Beauveria bassiana and deltametrin to resistant strains of Boophilus microplus. Annals of the New York Academy of Sciences, v.1026, n.1, p.319-322, 2004.

BARCI, L.A.G. Seleção de isolados do fungo Beauveria bassiana (Bals.) Vuill. para o controle de larvas do carrapato Boophilus microplus (Canestrini). 2007. 146f. Tese (Doutorado em Parasitologia) - Instituto de Biologia, Universidade Estadual de Campinas, Campinas, 2007.

CASTRO, J.J. de; NEWSON, R.M. Host resistance in cattle tick control. Parasitology Today, v.9, n.1, p.13-17, 1993.

CLEVELAND, C.B. Environmental and health assessments for spinosad against the backdrop of organic certification. ACS Symposium Series, v.947, p.109-130, 2007.

ER, M.K.; GÖKÇE, A. Effects of selected pesticides used against glasshouse tomato pests on colony growth and conidial germination of Paecilomyces fumosoroseus. Biological Control, v.31, p.398-404, 2004.

ESTAT - Sistema de análises estatísticas. Jaboticabal: Departamento de Ciências Exatas, FCAV/Unesp. V. 2.0 (livre), 1997. Disponível em: <http://www.fcav.unesp. $\mathrm{br} /$ download2/softweres/estat/. Online. Acesso em: 10 nov. 2009.

GHINI, R.; KIMATI, H. Resistência de fungos a fungicidas. Jaguariúna: Embrapa Meio Ambiente, 2000. 78p.

GRISI, L.; MASSARD, C.L.; MOYA BORJA, G.E.; PEREIRA, J.B. Impacto econômico das principais ectoparasitoses em bovinos no Brasil. A Hora Veterinária, v.21, n.125, p.8-10, 2002.

LOURENÇÃO, A.L;; KOMATSU, S.; ALVES, S.B. Controle de Sitophilus zeamais em milho com Beauveria 
bassiana, Metarhizium anisopliae e Pirimifos Metil. Ecossistema, v.18, n.1, p.69-74, 1993.

MARQUES, R.P.; MONTEIRO, A.C.; PEREIRA, G.T. Crescimento, esporulação e viabilidade de fungos entomopatogênicos em meios contendo diferentes concentrações do óleo de Nim (Azadirachta indica). Ciência Rural, v.34, n.6, p.1675-1680, 2004.

MONTEIRO, S.G.; MATIMOTO, L.R.; SILVEIRA, F.S.; LEAL, A.M. Isolamento de fungos em carrapatos ixodídeos naturalmente infectados. Revista da Faculdade de Zootecnia, Veterinária e Agronomia, v.10, n.1, p.65-71, 2003.

OLIVEIRA, R.C.; NEVES, P.M.O.J.; GUZZO, E.C.; ALVES, V.S. Compatibilidade de fungos entomopatogênicos com agroquímicos. Semina: ciências agrárias, v.23, n.2, p.211-216, 2002.

PACHAMUTHU, P.; KAMBLE, S.T.; YUEN, G.Y. Virulence of Metarhizium anisopliae (Deuteromycotina: Hyphomycetes) strain ESC-1 to the German cockroach (Dictyoptera: Blatellidae) and it's compatibility with insecticides. Journal of Economic Entomology, v.92, n.2, p.340-346, 1999.

PAIÃO, J.V.C. Compatibilidade dos fungos Beauveria bassiana e Metarhizium anisopliae com carrapaticidas quí- micos utilizados no controle de Boophilus microplus (Acari: Ixodidae). 2000. 55f. Dissertação (Mestrado em Microbiologia) - Programa de Pós-graduação em Microbiologia, Faculdade de Ciência Agrárias e Veterinárias, Universidade Estadual Paulista, Jaboticabal, 2000.

SAMISH, M.; GINDIN, G.; ALEKSEEV, E.; GLAZER, I. Pathogenicity of entomopathogenic fungi to different developmental stages of Rhiphicephalus sanguineus (Acari: Ixodidae). Journal of Parasitology, v.87, n.6, p.1355-1359, 2001.

WENZEL, I.M.; BATISTA FILHO, A.; ALMEIDA, A.M.B.; MINEIRO, J.L.C. Compatibilidade de Lecanicillium lecanii (Hyphomicetes), em condições de laboratório e estufa, aos agrotóxicos utilizados na cultura do crisântemo. Arquivos do Instituto Biológico, São Paulo, v.75, n.2, p.157166, 2004.

ZHIOUA, E.; HEYER, K.; BROWNING, M.; GINSBERG, H.S.; LEBRUN, R.A. Pathogenicity of Bacillus thuringiensis variety Kurstaki to Ixodes scapularis (Acari: Ixodidae). Journal of Medical Entomology, v.36, n.6, p.900-902, 1999.

Recebido em 16/12/09

Aceito em 15/4/11 